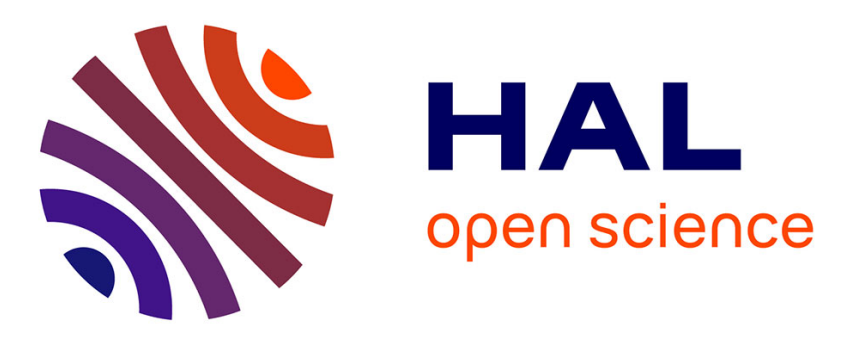

\title{
Centralized collaborative compressed sensing of wideband spectrum for cognitive radios
}

Hessam Moussavinik, Guibène Wael, Hayar Aawatif

\section{To cite this version:}

Hessam Moussavinik, Guibène Wael, Hayar Aawatif. Centralized collaborative compressed sensing of wideband spectrum for cognitive radios. IEEE Confrence on Ultra Modern Telecommunications and Control Systems and Workshops (ICUMT), 2010, Oct 2010, Moscou, Russia. pp.246 - 252. hal00689803

\section{HAL Id: hal-00689803 https://hal.science/hal-00689803}

Submitted on 20 Apr 2012

HAL is a multi-disciplinary open access archive for the deposit and dissemination of scientific research documents, whether they are published or not. The documents may come from teaching and research institutions in France or abroad, or from public or private research centers.
L'archive ouverte pluridisciplinaire HAL, est destinée au dépôt et à la diffusion de documents scientifiques de niveau recherche, publiés ou non, émanant des établissements d'enseignement et de recherche français ou étrangers, des laboratoires publics ou privés. 


\title{
Centralized Collaborative Compressed Sensing of Wideband Spectrum for Cognitive Radios
}

\author{
Hessam Moussavinik* Wael Guibène ${ }^{\dagger} \quad$ Aawatif Hayar $^{\dagger}$ \\ * Dept. of Electronics and Telecommunication, Norwegian University of Science and Technology, NTNU, N-7491 \\ Trondheim, Norway. \\ $\dagger$ EURECOM - Mobile Communication Dept., P.O. Box 193, 06904 Sophia Antipolis, France. \\ Email: Hessam.Moussavinik@iet.ntnu.no,Wael.Guibene@eurecom.fr, Aawatif.Hayar@eurecom.fr
}

\begin{abstract}
This paper ${ }^{(1)}$ presents a new centralized collaborative sensing technique for cognitive radio systems which combines algebraic tools and compressive sampling techniques. The proposed approach consists of the detection of spectrum holes using spectrum distribution discontinuities detector fed by compressed measurements. The compressed sensing algorithm is designed to take advantage from the primary signals sparsity and to keep the linearity and properties of the original signal in order to be able to apply algebraic detector on the compressed measurements. Collaboration among radios enables the cognitive network to detect hidden primary users and makes it more robust against fading and unknown channel conditions. Furthermore, as an important key point, collaboration makes it possible to sample more compressively at each radio, i.e., each radio performs sampling with a lower rate. The complexity of the proposed detector is also discussed and compared with the energy detector as reference algorithm. The comparison shows that the proposed technique outperforms energy detector in addition to its low complexity.
\end{abstract}

Index Terms-Collaborative compressed sensing, compressive sampling, cooperative, spectrum sensing, cognitive radio, distribution discontinuities, algebraic detection, wideband.

\section{INTORDUCTION}

Recently, compressed sensing/compressive sampling (CS) has been considered as a promising technique to improve and implement cognitive radio (CR) systems. Cognitive radio is an smart wireless communication system that is able to promote the efficiency of the spectrum usage by exploiting the free frequency bands in the spectrum, namely spectrum holes [1], [2]. Detection of spectrum holes is of the first steps of implementing a cognitive radio system. In wideband radio one may not be able to acquire a signal at the Nyquist sampling rate due to the current limitations in Analog-toDigital Converter (ADC) technology [3]. Compressive sensing makes it possible to reconstruct a sparse signal by taking less samples than Nyquist sampling, and thus wideband spectrum sensing is doable by CS. An sparse signal or a compressible signal is a signal that is essentially dependent on a number of degrees of freedom which is smaller than the dimension of the signal sampled at Nyquist rate. In general, signals of practical interest may be only nearly sparse [3].

(1) The research leading to these results has received funding from the European Community's Seventh Framework Programme (FP7/2007-2013) under grant agreement SACRA $\mathrm{n}^{\circ} 249060$, SENDORA, and the SAMPOS/WISENET Project, which is funded by the Research Council of Norway under the contract number 176875/S10 and 176724/I30.
Apart from reconstructing the original signal, detection is more required and interesting in the context of cognitive radio. Generally, for detection purposes it is not necessary to reconstruct the original signal, but only an estimate of the relevant sufficient statistics for the problem at hand is enough. This leads to less required measurements and lower computational complexity [4]. We do skip estimation of the original signal and instead we directly use the measurements for detection purpose, so the complexity of the system is reduced as much as possible.

It is known that a single cognitive radio may fail to detect a hidden node or primary user because of shadowing or being in deep fade. Collaboration among cognitive radios can greatly improve the detection performance of the cognitive network in such situations, and the probability that all radios be in deep fades becomes extremely low [5]. The other significant key point, which we are interested in, is that collaboration among radios reduces the sampling rate at each single radio that leads to less complexity and lower energy consumption at each radio. Lower sampling rate corresponds to less hardware complexity, and transmitting fewer samples to the fusion center corresponds to less energy consumption.

In this paper we develop a centralized implementation of collaborative compressed sensing of wideband spectrum for cognitive radios that is combined with distribution discontinuities detection technique. In this sense a group of cognitive radios identify spectrum holes of a wideband spectrum with low complexity while the sensing action is robust to severe fading environments.

The rest of the paper is organized as follows. Section II states the motivation and system model. Section III details the proposed collaborative compressed sensing for spectrum detection. Section IV is dedicated to performance results, and section $\mathrm{V}$ concludes this paper.

\section{Motivation And System Model}

\section{A. Motivation}

Cognitive radio (CR) as introduced by Joseph Mitola [6] is a self aware and intelligent device that can adapt itself to the wireless environment changes by first detecting them, and then adapting its radio parameters to the new opportunities. Cognitive radio technique is an smart wireless communication system that is able to promote the efficiency of the spectrum 
usage by exploiting the idle frequency bands in the spectrum, namely spectrum holes [1], [2]. Detection of spectrum holes is of the first steps of implementing a cognitive radio system. Different statistical approaches already exist. The easiest to implement and the reference one in terms of complexity is the energy detector (ED) [7]. Nevertheless, ED is highly sensitive to noise and does not perform well in low signal to noise ratio (SNR). Other advanced techniques based on signals modulations and exploiting some of the transmitted signals inner features were also developed [8]. For instance, the cyclostationary features detector (CFD) exploits the builtin cyclic properties of the PU received signal. The CFD has a great robustness to noise compared to ED but its high complexity is still a consequent drawback. Other techniques that were developed by researchers at Eurécom Institute are based on model selection tools and entropy investigation [9][11].

An important issue in spectrum sensing by cognitive radio is the complexity that is introduced by sampling rate of received signal at a CR. Specially when the spectrum to be sensed is wide the sampling is more challenging. In wideband radio one may not be able to acquire a signal at the Nyquist sampling rate due to the current limitations in Analog-to-Digital Converter (ADC) technology [3]. Also, applying the mechanism of narrowband detector for wideband spectrum sensing is inflexible and slow. Therefore, efficient wideband sensing techniques are highly demanded to increase the sensing agility [12].

Furthermore, collaborative sensing is generally required to detect hidden nodes to a $\mathrm{CR}$, that is several $\mathrm{CR}$ nodes cooperatively sense the spectrum to detect primary users (PUs) and available holes. In such scenarios, the amount of data processing at each CR node, in both centralized and decentralized schemes, and the amount of data exchange between $\mathrm{CR}$ nodes and the fusion center in the centralized scheme are important factors in complexity and power consumption of the system. Also, high number of samples, sampled at Nyquist rate, increase the power consumption and complexity at $\mathrm{CR}$ nodes.

In order to actualize sensing in wide spectrum and to reduce the complexity and power consumption at CR nodes, sampling at a smaller rate than Nyquist rate, while reconstruction or detection of signal is accurately possible, is a prominent key. Hence, compressive sampling or compressed sensing (CS) becomes a promising solution in realization of cognitive radio and reducing the complexity and power consumption. Compressive sampling enables us to do the sampling at a smaller rate than Nyquist rate, sometimes much smaller, and accurately reconstruct the sparse signal, or perform detection or estimation.

The first step for cognitive radio is to sense the spectrum and identify the spectrum holes, or in other words, detect the occupied frequency bands. Typically the wireless signal in open access networks is sparse in the frequency domain since depending on location and at some times the percentage of spectrum occupancy is low due to the idle or not deployed radios [1], [13]. For example, we can model the spectrally sparse wideband signals as

$$
s(t)=\sum_{j=0}^{N-1} \beta_{j} e^{i 2 \pi j t / N}, t=0, \cdots, N-1
$$

where $N$ is very large but the number of nonzero coefficients $\beta_{j}$ is much less than $N$. In this sense we can say that the signal is spectrally sparse [14]. Therefore, we would like to implement spectrum sensing in the context of cognitive radio by performing collaborative compressed sensing combined with distribution discontinuities detection. To avoid signal reconstruction burden we choose a sensing matrix that enables the algebraic detector properly works while accepting the compressed samples directly as input. Collaboration among cognitive radios greatly improve the sensing performance, while increasing the number of cooperative radios enables us for more reduction in the sampling rate at each individual radio, that is, more reduced complexity at each single radio.

\section{B. System Model}

We assume a centralized implementation where a group of collaborative cognitive radios are spread over a small area $\left(\sim 1 \mathrm{Km}^{2}\right)$ and each of the radios communicates with the fusion center through the control channel. For the radio channel between primary users and cognitive radios we assume flat fading with a Rayleigh distribution and shadowing effect. Here, we assume a perfect channel between radios and fusion center.

Let us consider a discrete representation of the received signal at a single radio given by:

$$
x(n)=A_{n} s(n)+e(n)
$$

where $A_{n}$ is modeling the channel, $s(n)$ represents the discrete signal, that is $s(t)$ sampled at Nyquist rate, and $e(n) \sim \mathcal{N}\left(0, \sigma^{2} I_{n}\right)$ is i.i.d. Gaussian noise where $I_{n}$ is an identity matrix of size $n$.

For the moment, assume only an individual radio that samples the received signal at Nyquist rate, that is, it observes $x(n)$. Then the radio sends its observations to the fusion center for being processed. We would like to distinguish between two classified hypothesis $\mathcal{H}_{0}$ and $\mathcal{H}_{1}$ :

$$
\begin{aligned}
& \mathcal{H}_{0}: \quad x(n)=e(n) \\
& \mathcal{H}_{1}: \quad x(n)=A_{n} s(n)+e(n)
\end{aligned}
$$

where $\mathcal{H}_{0}$ means that the sensed frequency band is white containing only noise and $\mathcal{H}_{1}$ means that the sensed frequency band is occupied with a signal corrupted by noise. The key parameter of all spectrum sensing algorithms are the false alarm probability $P_{F}$ and the detection probability $P_{D} \cdot P_{F}$ is the probability to determine a frequency band as occupied while it is free, thus $P_{F}$ should be kept as small as possible.

$$
P_{F}=P\left(\mathcal{H}_{1} \mid \mathcal{H}_{0}\right)=P\left(\text { a signal (user) is present } \mid \mathcal{H}_{0}\right)
$$

$P_{D}$ is the probability to determine a frequency band as occupied when there exists a signal, thus $P_{D}$ should be kept as large as possible.

$$
\begin{gathered}
P_{D}=1-P_{M}= \\
1-P\left(\mathcal{H}_{0} \mid \mathcal{H}_{1}\right)=1-P\left(\text { no signal (user) is present } \mid \mathcal{H}_{1}\right)
\end{gathered}
$$


where $P_{M}$ denote the probability of missed detection. To design the optimal detector on Neyman-Pearson criterion, we try to maximize the overall $P_{D}$ under a given overall $P_{F}$. In order to infer on the nature of the received signal, we calculate a threshold for each of the detectors. The decision threshold is determined using the required probability of false alarm $P_{F}$ given by (5). The threshold $T h$ for a given $P_{F}$ is determined by solving the equation:

$$
\left.P_{F}=P\left(\text { a signal is present } \mid \mathcal{H}_{0}\right)\right)=1-F_{\mathcal{H}_{0}}(T h)
$$

where $F_{\mathcal{H}_{0}}$ denote the cumulative distribution function (CDF) under $\mathcal{H}_{0}$.

The algebraic approach is able to detect the signal distribution discontinuities and find their positions in the spectrum, having the complete signal (Nyquist rate samples) as input to the detector. But as we previously mentioned, the problem is that sampling a wideband signal with Nyquist rate is constrained due to the reasons highlighted in section I. In order to make the detection possible with less number of samples or smaller sampling rate, relatively to Nyquist rate, we implement compressed sensing technique. In this sense, by considering the sparseness of the signal, at each individual radio we observe the received signal compressively with a smaller rate than Nyquist rate such as:

$$
y=\Phi x+e
$$

where $y \in \mathbb{R}^{M}$ is the compressed measurements or observations, $\Phi$ is the sensing matrix, $x \in \mathbb{R}^{N}$ is the received signal like $A_{n} s(n)$ as above, $e$ is the additive noise, and $M \ll N$. It is shown that with some conditions on $\Phi$ it is possible to recover $x$ accurately based on $y$.

We would like to use the compressed samples directly to detect the frequency holes without recovering the signal itself. Since the algebraic detection of distribution discontinuities is a linear approach we find a proper sensing matrix that makes it possible to use the compressed samples as the input to the linear detector. Cognitive radios can use a same sensing matrix or different sensing matrices of each other. Here, we assume that radios use different sensing matrices of each other that are made randomly as explained in following section.

After compressive sensing, performed by each individual radio, each radio sends the compressed observations to the fusion center. It is clear that sending the compressed observations requires lower energy and smaller bandwidth for the control channel than the case with Nyquist sampling. In following section we discuss about compressed sensing and the proper type of the sensing matrix as well as the detection process at the fusion center.

\section{Collaborative Compressed Sensing FOR SPECTRUM DETECTION}

\section{A. Compressive Sampling at an Individual Radio}

First, let us give an introduction on compressive sampling. Let $x \in \mathbb{R}^{N}$ be a signal with expansion in an orthonormal basis $\Psi$ as

$$
x(t)=\sum_{j=0}^{N-1} \alpha_{j} \psi_{j}(t), \quad t=0, \cdots, N-1
$$

where $\Psi$ is the $N \times N$ matrix with the waveforms $\psi_{j}$ as rows. To use convenient matrix notations we can write the decomposition as $x=\Psi \alpha$ or equivalently, $\alpha=\Psi^{*} x$ where $\Psi^{*}$ denotes conjugate transpose of $\Psi$. A signal $x$ is sparse in the $\Psi$ basis if the coefficient sequence $\alpha$ is supported on a small set. We say that a vector $\alpha$ is $S$-sparse if its support $\left\{j: \alpha_{j} \neq 0\right\}$ is of cardinality less or equal to $S$ [3]. Consider that we would like to recover all the $N$ coefficients of $x$, vector $\alpha$, from measurements $y$ about $x$ of the form

$$
y_{m}=\left\langle x, \phi_{m}\right\rangle=\sum_{n=0}^{N-1} \phi_{m n} x[n], m=0, \cdots, M-1
$$

or

$$
y=\Phi x=\Phi \Psi \alpha=\Theta \alpha
$$

where we are interested in the case that $M \ll N$, and the rows of the $M \times N$ sensing matrix $\Phi$ are incoherent with the columns of $\Psi$. Then it is shown that signal $x$ can accurately and sometimes exactly be recovered, considering that the recovered signal $x^{\star}$ is given by $x^{\star}=\Psi \alpha^{\star}$, and $\alpha^{\star}$ is the solution to the convex optimization program

$$
\min _{\tilde{\alpha} \in \mathbb{R}^{N}}\|\tilde{\alpha}\|_{l_{1}} \text { subject to } \Phi \Psi \tilde{\alpha}=\Theta \tilde{\alpha}=y
$$

where $\|\tilde{\alpha}\|_{l_{1}}:=\sum_{j=1}^{N}\left|\tilde{\alpha}_{j}\right|$. The compressed sensing (CS) theory states that there exists a measuring factor $c>1$ such that only $M:=c S$ incoherent measurements $y$ are needed to recover $x$ with high probability. We also have to mention that except $l_{1}$-minimization solution other methods such as greedy algorithms in [15] exist for recovering the sparse signal [3], [4], [14], [16]-[18].

In case of noisy measurements, i.e., $y=\Phi x+e$, where $e$ is noise with $\|e\|_{l_{2}} \leq \epsilon$, [18] shows that solution to

$$
\min _{\tilde{\alpha} \in \mathbb{R}^{N}}\|\tilde{\alpha}\|_{l_{1}} \text { subject to }\|\Theta \tilde{\alpha}-y\|_{l_{2}} \leq \epsilon
$$

recovers the sparse signal with an error at most proportional to the noise level. Also, [18] discuss the conditions for stable recovery from noisy measurements.

Each single radio sends the compressed observations to the fusion center. In the fusion center the observations from each radio are processed separately by an algebraic approach to produce the detection result from each radio. Later, we explain how the detection results from all radios make the final detection of spectrum holes.

We are interested in doing the spectrum holes detection using algebraic approach directly from the compressed measurements without reconstructing the original signal itself. For this reason we must find out the appropriate sensing matrix according to the detection technique. The proposed detection technique is a linear algebraic algorithm. This technique uses the Fourier transform of the observed signal to detect the occupied frequency bands in the observed spectrum. Therefore the compressed measurements of the observed signal must keep the linearity and properties of the original signal in order to apply the detection algorithm successfully on the compressed measurements. In [19] we show that any row vector of the sensing matrix is a Dirac function, that is, only one column of each row is nonzero. To generate the sensing 
matrix we can start from generating $\Phi^{T}$ matrix by randomly selecting $M$ columns of an identity matrix $I_{N}$. The sensing matrix, $\Phi$, is given by transpose of $\Phi^{T}$, where the columns of the sensing matrix are unit-normed. So the sensing matrix $\Phi$ that we apply has a form like this

$$
\Phi \sim\left[\begin{array}{cccccccc}
0 & 1 & 0 & \cdots & 0 & 0 & 0 & 0 \\
\vdots & \vdots & \vdots & \vdots & \vdots & \vdots & \vdots & \vdots \\
0 & 0 & 0 & \cdots & 0 & 1 & 0 & 0
\end{array}\right]_{M \times N} .
$$

This form of sensing matrix gives us the opportunity to use the compressed measurements from each radio directly as input to the algebraic detection algorithm in the fusion center and thus avoiding the computation complexity of reconstructing the original signal. Radios can have a similar sensing matrix, but here we use a different random sensing matrix for each radio. Following, detection process at the fusion center is explained.

\section{B. Centralized Detection at the Fusion Center}

As we said, the compressed observations from each radio is processed separately at the fusion center with an algebraic approach. The algebraic detection (AD) is a new approach based on advanced differential algebra and operational calculus. In this method, the primary user's presence is rather casted as a change point detection in its transmission spectrum [20]. In this approach, the mathematical representation of the spectrum of the compressed measurements, i.e., the observed signal $Y_{n}$ from each radio in frequency domain, is assumed to be a piecewise $P^{t h}$ polynomial signal expressed as following:

$$
Y_{n}=\sum_{k=1}^{K} \mathcal{Y}_{k}\left[n_{k-1}, n_{k}\right](f) p_{k}\left(n-n_{k-1}\right)+E_{n}
$$

where $\mathcal{Y}_{k}\left[n_{k-1}, n_{k}\right]$ is the characteristic function, $p_{k}$ is a polynomial series of order $P, E_{n}$ is the additive corrupting noise, $K$ is the number of subbands defined in the frequency range of observation interest, and $n=\frac{f}{f_{s}}$ is the normalized frequency, where $f_{s}$ is the sampling frequency and $f$ is the signal frequency.

Let us define the clean version of the received signal $S_{n}$ as:

$$
S_{n}=\sum_{k=1}^{K} \mathcal{Y}_{k}\left[n_{k-1}, n_{k}\right](f) p_{k}\left(n-n_{k-1}\right)
$$

And let $b$, the frequency band, is such that one and only one change point occurs in the interval $I_{b}=\left[n_{k-1}, n_{k}\right]=[\nu, \nu+b]$, $\nu \geq 0$. Denoting $S_{\nu}(n)=S(n+\nu), n \in[0, b]$ as the restriction of the signal in the interval $I_{b}$ and redefine the change point $n_{\nu}$ relatively to $I_{b}$ such as:

$$
\begin{cases}n_{\nu}=0 & \text { if } S_{\nu} \text { is continuous } \\ 0<n_{\nu} \leq b & \text { otherwise }\end{cases}
$$

Then, the primary user presence on a sensed sub-band is equivalent to find $0<n_{\nu} \leq b$ on that band. The $\mathrm{AD}$ gives the opportunity to build a whole family of spectrum sensing detectors, depending on a given model order $P$. Depending on this model order, we can show that performance of the AD is increasing as the order $P$ increases.

The proposed algorithm is implemented as a filter bank which composed of $P$ filters mounted in a parallel way. The impulse response of each filter is:

$$
h_{k+1, n}=\left\{\begin{array}{l}
\frac{\left(n^{l}(b-n)^{P+k}\right)^{(k)}}{(l-1) !}, 0<n<b \\
0, \text { otherwise }
\end{array}\right.
$$

where $k \in[0 \cdots P-1]$ and $l$ is chosen such that $l>$ $2 \times P$. The proposed expression of $h_{k+1, n}, k \in[0 \cdots P-1]$ is determined by modeling the spectrum with a piecewise regular signal in frequency domain and casting the problem of spectrum sensing as a change point detection in the primary user transmission [20]. Finally, in each detected interval $\left[n_{\nu_{i}}, n_{\nu_{i+1}}\right]$, we compute the following equation:

$$
\lambda_{k+1}=\sum_{m=n_{\nu_{i}}}^{n_{\nu_{i+1}}} W_{m} h_{k+1, m} X_{m}
$$

where $W_{m}$ is the weight for numeric integration defined by:

$$
\left\{\begin{array}{l}
W_{0}=W_{M}=0.5 \\
W_{m}=1 \text { otherwise }
\end{array}\right.
$$

and $M$ is the number of samples of the observed signal.

In order to infer whether the primary user is present in its sub-band, a decision function is computed as following:

$$
D_{f}=\left\|\prod_{k=0}^{P} \lambda_{k+1}\left(n_{\nu}\right)\right\|
$$

The decision is made by comparing the threshold $T h$ to the mean value of the decision function over the detected intervals.

In the collaborative sensing the final decision is made by applying a rule on the decisions from all the radios for each detected interval. Different rules maybe used, and here, we choose the averaging rule. Let us denote the detection results from collaboration by $\mathcal{D}$. Decision function from each radio $\rho$ on each interval $\left[\nu_{i}, \nu_{i+1}\right]$ is denoted by $D_{f_{\rho, \nu_{i}}}$. Then we have:

$$
\mathcal{D}_{\nu}=\frac{1}{R s} \sum_{\rho=1}^{R s} D_{f_{\rho, \nu_{i}}}
$$

where $R s$ is the number of collaborative radios. So, the final decision is made by comparing $\mathcal{D}$ to the threshold $T h$ over detected subbands. In next section simulation results on the proposed technique are presented.

\section{Simulations}

In this section we investigate the performance of the proposed collaborative compressed sensing with the energy detector (ED). ED is the most common method for spectrum sensing because of its non-coherency and low complexity. The energy detector measures the received energy during a finite time interval and compares it to a predetermined threshold. That is, the test statistic of the energy detector is:

$$
\sum_{m=1}^{M}\left\|y_{m}\right\|^{2}
$$

where $M$ is the number of samples of the received signal $y$ from each radio. 
Traditional ED can be simply implemented as a spectrum analyzer. A threshold used for primary user detection is highly susceptible to unknown or changing noise levels. Even if the threshold would be set adaptively, presence of any in-band interference would confuse the energy detector.

Since the complexity of sensing algorithms is a major concern in implementation and ED is well known for its simplicity, we choose ED as the comparison reference. Denoting $N$ the number of Nyquist samples of the observed signal $y$ and $P$ the model order of AD, we show that the complexity of $\mathrm{AD}$ is $P N$ and the complexity of ED is $N$. From these results, we clearly see that the exploited sensing algorithm has a comparable complexity to the energy detector. For the proposed AD based compressed sensing algorithm, the complexity is equal to: $P \frac{M}{N} N=P M$, where $M$ is the number of compressed measurements of the received signal and $M \ll N$.

Table I summarizes the complexity of each detector.

\begin{tabular}{|l|c|}
\hline Sensing technique & Complexity \\
\hline Energy detector & $\mathrm{N}$ \\
Algebraic detector & $\mathrm{PN}$ \\
Combined compressive sampling and & $\mathrm{PM}$ \\
distribution discontinuities detection & \\
\hline
\end{tabular}

TABLE I

COMPLEXITY COMPARISON OF THE THREE SENSING TECHNIQUES; $M \ll N$.

In order to achieve realistic and well founded simulations, DVB-T signals based on DVB-T $2 \mathrm{~K}$ recommendations are used as the signals to be sensed. This choice can be justified by the fact that almost all licensed primary networks are DVB-T and secondary users are CR deployed in these networks. The signal and channel parameters are given in Table II.

We consider collaborative radios group with size

\begin{tabular}{|l|l|}
\hline Bandwidth & $8 \mathrm{MHz}$ \\
\hline Mode & $2 \mathrm{~K}$ \\
\hline Guard interval & $1 / 4$ \\
\hline Channel models & AWGN \\
\hline Flat fading & Multipath \\
\hline Channel gains & {$[-10,0] \mathrm{dB}$} \\
\hline Sensing time & $1.25 \mathrm{~ms}$ \\
\hline
\end{tabular}

TABLE II

THE TRANSMITTED DVB-T PRIMARY USER SIGNAL AND CHANNEL PARAMETERS

of $1,5,10,20$ radios. For each group size, compressed collaborative detection with different compression ratios, $\frac{M}{N}=\{10 \%, 20 \%, 30 \%\}$, is simulated and compared to the ED detector with single radio and no compression. Collaborative ED is not simulated due to timing issues and since the comparison is still valid with single radio because, as will be seen, performance of a single radio with compressed sensing for compression ratios of about $20 \%$ and higher is better than single ED with no compression. So, we expect that cooperative compressed sensing also ourperforms cooperative ED, intuitively.

Figure 1 shows the performance of energy detector (ED) with no compression and first order algebraic detector $A D_{1}$ with different compression ratios for collaborative groups of size $1,5,10$ and 20 radios.

We note that only performance of a single radio with compression ratio of $10 \%$ is not as good as performance of
ED with single radio and no compression. And for the rest of examples the performance is better. This is where the complexity of the compressed sensing, i.e., $\frac{M}{N}$, is much lower than ED, i.e., $N$. Collaboration among radios greatly improve the detection performance. Also, we note that when the number of collaborations increases the compression ratio at each radio can be decreased in order to achieve a specific probability of detection $P_{D}$. Figure 2 shows the probability of detection that is achievable by different number of collaborative radios for a compressed sensing ratio of $\frac{M}{N}=10 \%$ at $\mathrm{SNR}=-20 \mathrm{~dB}$ and $P_{F}=0.05$.

These results are obtained with Algebraic detection of order $P=1$, where increasing the order to $P=2$ improves the performance dramatically, while complexity increases to $2 M$ from $M$. But, still, for example for $\frac{M}{N}=10 \%$, complexity remains much less than complexity of ED, i.e., $N$.

As a final word, we can say that the collaborative compressed sensing decreases the complexity and energy consumption of cognitive radio networks remarkably due to the low sampling rate required for each radio while it makes the cognitive network robust to fading.

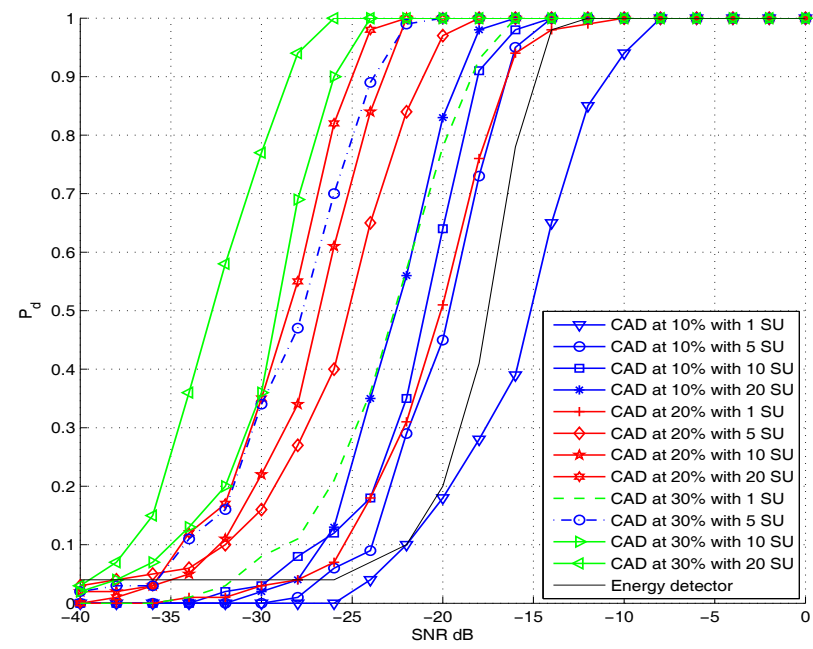

Fig. 1. Probability of detection, $P_{D}$, vs. SNR at $P_{F}=0.05$. CAD: Compressed sensing with Algebraic detection of order $P=1$. SU: secondary users/collaborative radios.

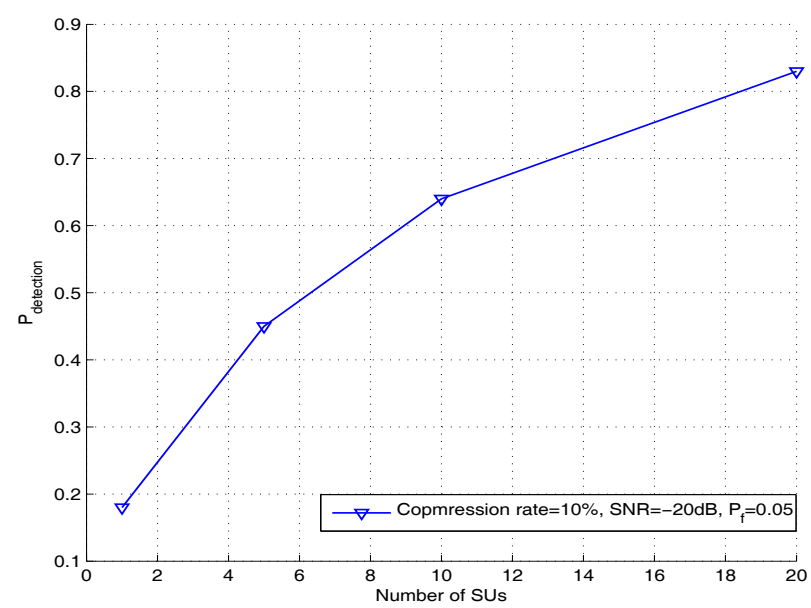

Fig. 2. Probability of detection, $P_{D}$, vs. number of collaborative radios (SUs: secondary users) with compressed sensing of ratio $\frac{M}{N}=10 \%$ and Algebraic detection of order $P=1$, at $\mathrm{SNR}=-20 \mathrm{~d} B$ and $P_{F}^{N}=0.05$. 


\section{CONCLUSION}

In this work we present a new centralized collaborative sensing technique which combines compressive sampling and algebraic method to detect spectrum holes. We choose a compressed sensing matrix which keeps the linear properties of the sampled primary signal. Each radio of the collaboration group use a different random sensing matrix and sends its compressed observations to the fusion center. The fusion center process the compressed measurements from each radio separately with an algebraic detector. The fusion center makes the final decision by averaging over results from all the collaborative radios to localize spectrum distribution discontinuities and identify spectrum holes. The performance comparison at different sampling rates shows that the new designed scheme achieves better performance than energy detector while preserving a low computational complexity. Also, as an important key point, by increasing the number of collaborative radios the sampling rate at each radio decreases, in order to obtain a desired performance. This leads to a great reduction in terms of system complexity and implementation.

\section{REFERENCES}

[1] S. Haykin, "Cognitive radio: brain-empowered wireless communications," Selected Areas in Communications, IEEE Journal on, vol. 23, no. 2, pp. 201-220, 2005.

[2] J. Mitola and G. Maguire, "Cognitive radio: making software radios more personal," Personal Communications, IEEE, vol. 6, no. 4, pp. 13$18,1999$.

[3] E. J. Cands, "Compressive sampling," in Proceedings of the International Congress of Mathematicians, (Madrid, Spain), 2006.

[4] M. Duarte, M. Davenport, M. Wakin, and R. Baraniuk, "Sparse signal detection from incoherent projections," in Acoustics, Speech and Signal Processing, 2006. ICASSP 2006 Proceedings. 2006 IEEE International Conference on, vol. 3, p. III, 2006.

[5] S. M. Mishra, A. Sahai, and R. W. Brodersen, "Cooperative sensing among cognitive radios," in Proc. of the IEEE International Conference on Communications (ICC), 2006.
[6] J. M. Iii, An Integrated Agent Architecture for Software Defined Radio. Doctor of technology, Royal Inst. Technol. (KTH), Stockholm, Sweden, 2000.

[7] H. Urkowitz, "Energy detection of unknown deterministic signals," Proceedings of the IEEE, vol. 55, no. 4, p. 523531, 1967.

[8] A. Sahai and D. Cabric, "A tutorial on spectrum sensing: fundamental limits and practical challenges," in Proc. IEEE Symposium on New Frontiers in Dynamic Spectrum Access Networks (DySPAN), Nov. 2005.

[9] B. Zayen, A. Hayar, and K. Kansanen, "Blind spectrum sensing for cognitive radio based on signal space dimension estimation," in IEEE International Conference on Communications, p. 1418, June 2009.

[10] B. Zayen, A. Hayar, and D. Nussbaum, "Blind spectrum sensing for cognitive radio based on model selection," in Cognitive Radio Oriented Wireless Networks and Communications, 2008. CrownCom 2008. 3rd International Conference on, p. 14, 2008.

[11] M. Haddad, A. M. Hayar, M. H. Fetoui, and M. Debbah, "Cognitive radio sensing information-theoretic criteria based," in Cognitive Radio Oriented Wireless Networks and Communications, 2007. CrownCom 2007. 2nd International Conference on, p. 241244, 2007.

[12] Z. Quan, S. Cui, H. V. Poor, and A. H. Sayed, "Collaborative wideband sensing for cognitive radios," IEEE Signal Processing Magazine, vol. 25, no. 6 , p. 6073,2008 .

[13] Z. Tian and G. Giannakis, "Compressed sensing for wideband cognitive radios," in Acoustics, Speech and Signal Processing, 2007. ICASSP 2007. IEEE International Conference on, vol. 4, pp. IV-1357-IV-1360, 2007.

[14] E. Candes and M. Wakin, "An introduction to compressive sampling," IEEE Signal Processing Magazine, vol. 25, no. 2, pp. 21-30, 2008.

[15] J. A. Tropp and A. C. Gilbert, "Signal recovery from random measurements via orthogonal matching pursuit," IEEE Transactions on Information Theory, vol. 53, no. 12, pp. 4655-4666, 2007.

[16] D. Donoho, "Compressed sensing," Information Theory, IEEE Transactions on, vol. 52, no. 4, pp. 1289-1306, 2006.

[17] E. Candes, J. Romberg, and T. Tao, "Robust uncertainty principles: exact signal reconstruction from highly incomplete frequency information," Information Theory, IEEE Transactions on, vol. 52, no. 2, pp. 489-509, 2006.

[18] E. Candes, J. Romberg, and T. Tao, "Stable signal recovery from incomplete and inaccurate measurements," math/0503066, Mar. 2005.

[19] A. Hayar, W. Guibene, and H. Moussavinik, "Combined compressive sampling and distribution discontinuities detection approach to wideband spectrum sensing for cognitive radios," Submitted to Journal of Communications and Networks, JCN, no. SPARSITY IN COMMUNICATIONS, 2010.

[20] W. GUIBENE, M. TURKI, A. HAYAR, and B. ZAYEN, "Development and implementation of an algebraic detector for spectrum sensing," submitted to IEEE Journal on Selected Areas in Communications: Special Issue on Advances in Cognitive Radio Networking and Communications. 